TAIWANESE JOURNAL OF MATHEMATICS

Vol. 11, No. 4, pp. 999-1018, September 2007

This paper is available online at http://www.math.nthu.edu.tw/tjm/

\title{
A CHARACTERIZATION OF DUAL LORENTZIAN SPHERICAL CURVES IN THE DUAL LORENTZIAN SPACE
}

\author{
Nihat Ayyıldız, A. Ceylan Çöken and Ahmet Yücesan
}

\begin{abstract}
A differential equation characterizing the dual Lorentzian spherical curves and an explicit solution of this differential equation are given. Also, without the precondition on the dual torsion is nowhere pure-dual, a necessary and sufficient condition for a curve to be dual Lorentzian spherical is presented. In addition, thanks to dual geodesic trihedron, torsion and curvature of dual Lorentzian curve $\hat{x}(s)$ have been calculated.
\end{abstract}

\section{INTRODUCTION}

Semi-Riemannian metric is quite important in differential geometry as well as in physics in which it plays a central role in the theory relativity especially as Lorentzian metric. Semi-Euclidean spaces $\mathbb{R}_{\nu}^{n+1}$ reduce to $\mathbb{R}^{n}$ if $\nu=0$. For $n \geq 1$, $\mathbb{R}_{1}^{n+1}$ is called Minkowski $(n+1)$-space; if $n=3$ it is the simplest example of a relativistic spacetime [10].

Minkowski geometry is a non-Euclidean geometry in a finite number of dimensions that is different from elliptic and hyperbolic geometry (and from the Minkowskian geometry of spacetime). Here the linear structure is the same as the Euclidean one but distance is not uniform in all directions. Instead of the usual sphere in Euclidean space, the unit ball is a general symmetric convex set. Therefore, although the parallel axiom is valid, Pythagoras theorem is not [12 ].

Theory of space curves of Riemannian manifold $M$ is fully developed and its local and global geometry is well-known. If $M$ is proper semi-Riemannian, there are three categories of curves, namely, spacelike, timelike and null, depending on their causal character [10].

Received June 8, 2005, accepted November 29, 2005.

Communicated by Chun-Lian Terng.

2000 Mathematics Subject Classification: 53C50.

Key words and phrases: Dual Lorentzian Space, Dual Lorentzian spherical curve, Spacelike-Timelike curve, Frenet vectors. 
The differential equation characterizing a real spherical curve is

$$
\frac{d}{d s}\left[\frac{1}{\tau(s)} \frac{d \rho}{d s}\right]+\rho(s) \tau(s)=0,
$$

where $s$ is the length of arc, $\rho(s)=\frac{1}{\varkappa(s)}$ is the radius of curvature and $\tau(s)$ is the torsion of the curve.

In the 1971, an explicit solution was first given by Breuer and Gottlieb as

$$
\rho(s)=a \cos \int_{0}^{s} \tau(s) d s+b \sin \int_{0}^{s} \tau(s) d s,
$$

where $a$ and $b$ are arbitrary constants.

A year later Wong observed that whereas the differential equation holds true only locally (for $\tau \neq 0$ ), the Breuer-Gottlieb solution holds regardless of a possible vanishing of $\tau$ at some point and is therefore a global characterization of a spherical curve.

Wong's observation was derived by using a global differential condition of his then a curve in $\mathbb{R}^{3}$ lies in a sphere iff there is a function $f \in C^{1}$ so that $f \tau=\rho^{\prime}$, $f^{\prime}=-\tau \rho[9]$.

This paper gives a characterization of dual Lorentzian spherical curves in the dual Lorentzian space.

\section{PReliminaries}

Let $\mathbb{R}^{3}=\left\{\left(x_{1}, x_{2}, x_{3}\right) \mid x_{1}, x_{2}, x_{3} \in \mathbb{R}\right\}$ be a 3 -dimensional vector space, $\vec{x}=\left(x_{1}, x_{2}, x_{3}\right)$ and $\vec{y}=\left(y_{1}, y_{2}, y_{3}\right)$ be two vectors in $\mathbb{R}^{3}$, the Lorentzian scalar product of $\vec{x}$ and $\vec{y}$ is defined by

$$
<\vec{x}, \vec{y}>=-x_{1} y_{1}+x_{2} y_{2}+x_{3} y_{3}
$$

$\left(\mathbb{R}^{3},<,>\right)$ is called 3-dimensional Lorentzian space, or Minkowski 3-space. We denote $\mathbb{R}_{1}^{3}$ as $\left(\mathbb{R}^{3},<,>\right)$.

For any $\vec{x}=\left(x_{1}, x_{2}, x_{3}\right), \vec{y}=\left(y_{1}, y_{2}, y_{3}\right) \in \mathbb{R}_{1}^{3}$, the Lorentzian vector product of $\vec{x}$ and $\vec{y}$ is defined by

$$
\vec{x} \wedge \vec{y}=\left(x_{2} y_{3}-x_{3} y_{2}, x_{1} y_{3}-x_{3} y_{1}, x_{2} y_{1}-x_{1} y_{2}\right),
$$

where $\overrightarrow{e_{1}} \wedge \overrightarrow{e_{2}}=-\overrightarrow{e_{3}}, \overrightarrow{e_{2}} \wedge \overrightarrow{e_{3}}=\overrightarrow{e_{1}}$ and $\overrightarrow{e_{3}} \wedge \overrightarrow{e_{1}}=-\overrightarrow{e_{2}}[1]$.

Moreover $\vec{x}$ in $\mathbb{R}_{1}^{3}$ is called a spacelike vector, a lightlike vector or a timelike vector if $<\vec{x}, \vec{x}>>0$, or $\vec{x}=\overrightarrow{0},<\vec{x}, \vec{x}>=0$ and $<\vec{x} \neq \overrightarrow{0}>$ or $<\vec{x}, \vec{x}><0$, 
respectively. For $\vec{x} \in \mathbb{R}_{1}^{3}$, the norm of $\vec{x}$ is defined by $\|\vec{x}\|=\sqrt{|<\vec{x}, \vec{x}\rangle \mid}$, and $\vec{x}$ is called a unit vector if $\|\vec{x}\|=1[10]$.

Let

$$
\begin{aligned}
\alpha: I \subset \mathbb{R} & \rightarrow \mathbb{R}_{1}^{3} \\
t & \rightarrow \alpha(t)=\left(\alpha_{1}(t), \alpha_{2}(t), \alpha_{3}(t)\right)
\end{aligned}
$$

be a smooth regular curve in $\mathbb{R}_{1}^{3}$, where $I$ is an open interval. For any $t \in I$, the curve $\alpha$ is called a spacelike curve, a lightlike curve or a timelike curve if $<\vec{\alpha}^{\prime}, \vec{\alpha}^{\prime}>>0,<\vec{\alpha}^{\prime}, \vec{\alpha}^{\prime}>=0$ or $<\vec{\alpha}^{\prime}, \vec{\alpha}^{\prime}><0$ respectively [10].

The Lorentzian and hyperbolic spheres with radius 1 in $\mathbb{R}_{1}^{3}$ are defined by

$$
S_{1}^{2}=\left\{\vec{x}=\left(x_{1}, x_{2}, x_{3}\right) \in \mathbb{R}_{1}^{3} \mid<\vec{x}, \vec{x}>=1\right\},
$$

and

$$
H_{0}^{2}=\left\{\vec{x}=\left(x_{1}, x_{2}, x_{3}\right) \in \mathbb{R}_{1}^{3} \mid<\vec{x}, \vec{x}>=-1\right\},
$$

respectively [10].

Dual numbers had been introduced by W.K. Clifford $(1849-1879)$ as a tool for his geometrical investigations. After him E.Study used dual numbers and dual vectors in his research on line geometry and kinematics. He devoted special attention to the representation of oriented lines by dual unit vectors and defined the famous mapping: The set of oriented lines in a Euclidean three-dimension space $\mathbb{R}^{3}$ is one to one correspondence with the points of a dual space $\mathbb{D}^{3}$ of triples of dual numbers [8].

Definition 2.1. By a dual number $\hat{x}$, we mean an ordered pairs of the form $\left(x, x_{0}\right)$ for $\forall x, x_{0} \in \mathbb{R}$. Let the set $\mathbb{R} \times \mathbb{R}$ be demonstrated as $\mathbb{D}$. Two inner operations and an equality on $\mathbb{D}=\left\{\left(x, x_{0}\right) \mid x, x_{0} \in \mathbb{R}\right\}$ are defined as follows:

(i) Inner production $\oplus: \mathbb{D} \times \mathbb{D} \rightarrow \mathbb{D}$ for $\hat{x}=\left(x, x_{0}\right), \hat{y}=\left(y, y_{0}\right)$ is defined as

$$
\hat{x} \oplus \hat{y}=\left(x, x_{0}\right) \oplus\left(y, y_{0}\right)=\left(x+y, x_{0}+y_{0}\right)
$$

and is called the addition in $\mathbb{D}$.

(ii) Inner production $\odot: \mathbb{D} \times \mathbb{D} \rightarrow \mathbb{D}$ for $\hat{x}=\left(x, x_{0}\right), \hat{y}=\left(y, y_{0}\right)$ is defined as

$$
\hat{x} \odot \hat{y}=\hat{x} \hat{y}=\left(x, x_{0}\right) \odot\left(y, y_{0}\right)=\left(x y, x y_{0}+x_{0} y\right)
$$

and is called the multiplication in $\mathbb{D}$.

(iii) If $x=y, x_{0}=y_{0}$ for $\hat{x}=\left(x, x_{0}\right), \hat{y}=\left(y, y_{0}\right) \in \mathbb{D}, \hat{x}$ and $\hat{y}$ are equal and it is indicated as $\hat{x}=\hat{y}$. 
Definition 2.2. If the operations of addition, multiplication and equality on the set $\mathbb{D}=\mathbb{R} \times \mathbb{R}$ with set of real numbers $\mathbb{R}$ are defined as above, the set $\mathbb{D}$ is called the dual numbers system and the element $\forall\left(x, x_{0}\right) \in \mathbb{D}$ is called a dual number.

Definition 2.3. In a dual number $\hat{x}=\left(x, x_{0}\right) \in \mathbb{D}$, the real number $x$ is called the real part of $\hat{x}$ and the real number $x_{0}$ is called the dual part of $\hat{x}$.

Definition 2.4. The dual number $(1,0)=1$ is called unit element of multiplication operation in $\mathbb{D}$ or real unit in $\mathbb{D}$.

Definition 2.5. The dual number $(0,1)$ is to be demonstrated with $\xi$ in short, and the $(0,1)=\xi$ is to be called dual unit. In accordance with the definition of the operation of multiplication, it can easily be seen that $\xi^{2}=0$. Also, the dual number $\hat{x}=\left(x, x_{0}\right) \in \mathbb{D}$ can be written as $\hat{x}=x+\xi x_{0}[7]$.

The set of $\mathbb{D}=\left\{\hat{x}=x+\xi x_{0} \mid x, x_{0} \in \mathbb{R}\right\}$ of dual numbers is a commutative ring according to the operations

(i) $\left(x+\xi x_{0}\right) \oplus\left(y+\xi y_{0}\right)=(x+y)+\xi\left(x_{0}+y_{0}\right)$

(ii) $\left(x+\xi x_{0}\right) \odot\left(y+\xi y_{0}\right)=x y+\xi\left(x y_{0}+y x_{0}\right)$.

The dual number $\hat{x}=x+\xi x_{0}$ divided by the dual number $\widehat{y}=y+\xi y_{0}$ providing $y \neq 0$ can be defined as

$$
\frac{\hat{x}}{\hat{y}}=\frac{x+\xi x_{0}}{y+\xi y_{0}}=\frac{x}{y}+\xi \frac{x_{0} y-x y_{0}}{y^{2}} .
$$

The set of

$$
\begin{aligned}
\mathbb{D}^{3}=\mathbb{D} \times \mathbb{D} \times \mathbb{D} & =\left\{\vec{x} \mid \overrightarrow{\hat{x}}=\left(x_{1}+\xi x_{0_{1}}, x_{2}+\xi x_{0_{2}}, x_{3}+\xi x_{0_{3}}\right)\right. \\
& =\left(x_{1}, x_{2}, x_{3}\right)+\xi\left(x_{0_{1}}, x_{0_{2}}, x_{0_{3}}\right) \\
& \left.=\vec{x}+\xi \overrightarrow{x_{0}}, \quad \vec{x} \in \mathbb{R}^{3}, \overrightarrow{x_{0}} \in \mathbb{R}^{3}\right\}
\end{aligned}
$$

is a modul on the ring $\mathbb{D}$.

Definition 2.6. Let $\vec{x}=\vec{x}+\xi \overrightarrow{x_{0}}, \vec{y}=\vec{y}+\xi \overrightarrow{y_{0}} \in \mathbb{D}^{3}$. The Lorentzian inner product of $\vec{x}$ and $\vec{y}$ is defined by

$$
<\overrightarrow{\hat{x}}, \vec{y}>=<\vec{x}, \vec{y}>+\xi\left(<\vec{x}, \overrightarrow{y_{0}}>+<\overrightarrow{x_{0}}, \vec{y}>\right) .
$$

We call the dual space $\mathbb{D}^{3}$ together with this Lorentzian inner product as dual Lorentzian space and indicate it by $\mathbb{D}_{1}^{3}[14]$.

Definition 2.7. Let $\vec{x}=\vec{x}+\xi \overrightarrow{x_{0}} \in \mathbb{D}_{1}^{3}$. $\vec{x}$ is said to be spacelike, timelike and lightlike (null) if the vector $\vec{x}$ is spacelike, timelike and lightlike (null), respectively. 
If $\vec{x} \neq 0$, the norm $\|\overrightarrow{\hat{x}}\|$ of $\overrightarrow{\hat{x}}=\vec{x}+\xi \overrightarrow{x_{0}}$ is defined by

$$
\|\overrightarrow{\hat{x}}\|=\sqrt{|<\overrightarrow{\hat{x}}, \overrightarrow{\hat{x}}>|}=\left(\|\vec{x}\|, \frac{<\vec{x}, \overrightarrow{x_{0}}>}{\|\vec{x}\|}\right) .
$$

A dual Lorentzian vector $\vec{x}$ with norm 1 is called a dual Lorentzian unit vector. It follows that $\overrightarrow{\hat{x}}$ is a dual timelike unit vector (resp., dual spacelike unit vector) if the relations $\left\langle\vec{x}, \vec{x}>=-1\right.$, (resp., $<\vec{x}, \vec{x}>=1$ ) and $\left\langle\vec{x}, \overrightarrow{x_{0}}>=0\right.$ hold [14].

Definition 2.8. Let $\vec{x}=\vec{x}+\xi \overrightarrow{x_{0}} \in \mathbb{D}_{1}^{3}$.

(i) The set $\hat{S}_{1}^{2}=\left\{\overrightarrow{\hat{x}}=\vec{x}+\xi \overrightarrow{x_{0}} \mid\|\overrightarrow{\hat{x}}-\overrightarrow{\hat{m}}\|=(1,0) ; \vec{x}, \overrightarrow{x_{0}} \in \mathbb{R}_{1}^{3}, \vec{x}\right.$ spacelike $\}$ is called the dual Lorentzian unit sphere with the center $\vec{m}$ in $\mathbb{D}_{1}^{3}$.

(ii) The set $\hat{H}_{0}^{2}=\left\{\overrightarrow{\hat{x}}=\vec{x}+\xi \overrightarrow{x_{0}} \mid\|\overrightarrow{\hat{x}}-\overrightarrow{\hat{m}}\|=(1,0) ; \vec{x}, \overrightarrow{x_{0}} \in \mathbb{R}_{1}^{3}, \quad \vec{x}\right.$ timelike $\}$ is called the dual hyperbolic unit sphere with the center $\overrightarrow{\hat{m}}$ in $\mathbb{D}_{1}^{3}$ [14].

If every $x_{i}(t)$ and $x_{0_{i}}(t), 1 \leq i \leq 3$, real valued functions are differentiable, the dual curve

$$
\begin{aligned}
& \hat{x}: I \rightarrow \underset{\mathbb{D}_{1}^{3}}{\rightarrow} \\
& t \rightarrow \vec{x}(t)=\left(x_{1}(t)+\xi x_{0_{1}}(t), x_{2}(t)+\xi x_{0_{2}}(t), x_{3}(t)+\xi x_{0_{3}}(t)\right) \\
& =\overrightarrow{x(t)}+\xi \overrightarrow{x_{0}}(t)
\end{aligned}
$$

in $\mathbb{D}_{1}^{3}$ is a differentiable. We call the real part $\overrightarrow{x(t)}$ as indicatrix of $\hat{x}(t)$. The dual arc length of the curve $\hat{x}(t)$ from $t_{1}$ to $t$ is defined as

$$
\hat{s}=\int_{t_{1}}^{t}\left\|\vec{x}(t){ }^{\prime}\right\| d t=\int_{t_{1}}^{t}\left\|\overrightarrow{x(t)} \vec{x}^{\prime}\right\| d t+\xi \int_{t_{1}}^{t}<\vec{t},{\overrightarrow{x_{0}}}^{\prime}>d t=s+\xi s_{0} .
$$

From now on we will take the arc length $s$ of $\overrightarrow{x(t)}$ as the parameter instead of $t$.

\section{Frenet Formulae}

Now, we will obtain equations relative to the derivatives of Frenet vectors throughout the curve in $\mathbb{D}_{1}^{3}$. Let

$$
\begin{aligned}
\hat{x}: I & \rightarrow \mathbb{D}_{1}^{3} \\
s & \rightarrow \hat{x}(s)=x \overrightarrow{(s)}+\xi x_{0}(s)
\end{aligned}
$$


be a $C^{4}$ curve with the arc length parameter $s$ of the indicatrix. Then,

$$
\frac{d \overrightarrow{\hat{x}}}{d \hat{s}}=\frac{d \overrightarrow{\hat{x}}}{d s} \cdot \frac{d s}{d \hat{s}}=\overrightarrow{\hat{t}}
$$

is called the unit tangent vector of $\hat{x}(s)$. By the aid of the equation (2.1), we have

$$
\hat{s}=s+\xi \int_{s_{1}}^{s}<\vec{t},{\overrightarrow{x_{0}}}^{\prime}>d s
$$

and from this $\frac{d \hat{s}}{d s}=1+\xi \Delta$, where the prime denotes differentiation with respect to the arc length $s$ of indicatrix and $\Delta=<\vec{t},{\overrightarrow{x_{0}}}^{\prime}>$. Since $\overrightarrow{\hat{t}}$ has constant length 1 , its differentiation with respect to $\hat{s}$, which is given by

$$
\frac{d \overrightarrow{\hat{t}}}{d \hat{s}}=\frac{d \overrightarrow{\hat{t}}}{d s} \cdot \frac{d s}{d \hat{s}}=\frac{d^{2} \overrightarrow{\hat{x}}}{d \hat{s}^{2}}=\hat{\varkappa} \overrightarrow{\hat{n}},
$$

measures the way the curve is turning in $\mathbb{D}_{1}^{3}$. The norm of the vector $\frac{d \overrightarrow{\hat{t}}}{d \hat{s}}$ is called curvature function of $\hat{x}(s)$. We impose the restriction that the function $\hat{\varkappa}: I \rightarrow \mathbb{D}$ is never pure dual. Then, the unit vector $\overrightarrow{\hat{n}}=\frac{1}{\hat{\varkappa}} \frac{d \overrightarrow{\hat{t}}}{d \hat{s}}$ is called the normal of $\hat{x}(s)$. The vector $\overrightarrow{\hat{b}}$ is called the binormal of $\hat{x}(s)$. We call the vectors $\overrightarrow{\hat{t}}, \overrightarrow{\hat{n}}, \overrightarrow{\hat{b}}$ Frenet trihedron of $\hat{x}(s)$ at the point $\hat{x}(s)$. The equalities relative to derivatives of Frenet vectors $\overrightarrow{\hat{t}}, \overrightarrow{\hat{n}}, \overrightarrow{\hat{b}}$ throughout the curve are written in the matrix form

$$
\frac{d}{d \hat{s}}\left[\begin{array}{c}
\overrightarrow{\hat{t}} \\
\overrightarrow{\hat{n}} \\
\overrightarrow{\hat{b}}
\end{array}\right]=\left[\begin{array}{ccc}
0 & \hat{\varkappa} & 0 \\
-\varepsilon_{\vec{t}} \varepsilon_{\overrightarrow{\hat{n}}} \hat{\varkappa} & 0 & \hat{\tau} \\
0 & -\varepsilon_{\overrightarrow{\hat{n}}} \varepsilon_{\overrightarrow{\hat{b}}} \hat{\tau} & 0
\end{array}\right]\left[\begin{array}{c}
\overrightarrow{\hat{t}} \\
\overrightarrow{\hat{n}} \\
\overrightarrow{\hat{b}}
\end{array}\right]
$$

such that $<\overrightarrow{\hat{t}}, \overrightarrow{\hat{t}}>=\varepsilon_{\overrightarrow{\hat{t}}},<\overrightarrow{\hat{n}}, \overrightarrow{\hat{n}}>=\varepsilon_{\overrightarrow{\hat{n}}}, \quad<\overrightarrow{\hat{b}}, \overrightarrow{\hat{b}}>=\varepsilon_{\overrightarrow{\hat{b}}}$, and $<\overrightarrow{\hat{t}}, \overrightarrow{\hat{n}}>=<\overrightarrow{\hat{t}}, \overrightarrow{\hat{b}}>=<\overrightarrow{\hat{b}}, \overrightarrow{\hat{n}}>=0$. The function $\hat{\tau}: I \rightarrow \mathbb{D}$ is called the torsion of $\hat{x}(s)$. The formulae (3.1) are called the Frenet formulae. 


\section{Dual Lorentzian Osculating Sphere and Dual Lorentzian Spherical CURves}

The geometric place of the dual Lorentzian spheres with 3-contact points with $\hat{x}(s)$ in the point $\hat{x}(s)$ of the curve $\hat{x}(s) \in \mathbb{D}_{1}^{3}$ can be given by the following theorem.

Theorem 4.1. Let the curve $\hat{x}(s) \subset \mathbb{D}_{1}^{3}$ be given with the coordinate neighbourhood $(I, \hat{x})$. Let the Frenet trihedron in the point $\hat{x}(s)$ corresponding to $s \in I$ be $\{\overrightarrow{\hat{t}}, \overrightarrow{\hat{n}}, \overrightarrow{\hat{b}}\}$. The geometric place of the dual Lorentzian spheres with 3-contact points with $\hat{x}(s)$ in the point $\hat{x}(s)$ of the curve $\hat{x}(s) \in \mathbb{D}_{1}^{3}$ is

$$
\overrightarrow{\hat{m}}=\overrightarrow{\hat{x}}-\hat{m}_{2} \overrightarrow{\hat{n}}-\hat{m}_{3} \overrightarrow{\hat{b}} .
$$

Proof. Let the coordinate neighbourhood $(I, \hat{x})$ be given as the arc-length parameter for $\hat{x}(s)$. Let, in the point $\hat{x}(s)$, the center of the dual Lorentzian spheres with 3-contact points with $\hat{x}(s)$ be $\overrightarrow{\hat{m}}$ and the radius be $\hat{r}$. Accordingly, let us take the function

$$
\begin{aligned}
\hat{h}: I & \rightarrow \mathbb{D} \\
s & \rightarrow \hat{h}(s)=|<\overrightarrow{\hat{x}}-\overrightarrow{\hat{m}}, \overrightarrow{\hat{x}}-\overrightarrow{\hat{m}}>|-\hat{r}^{2}
\end{aligned}
$$

into consideration. For the curve $\vec{x}(s)$ to have 3 -contact points with the spheres of

$$
\hat{S}_{1}^{2}=\left\{\overrightarrow{\hat{x}}=\vec{x}+\xi \overrightarrow{x_{0}} \mid\|\overrightarrow{\hat{x}}-\overrightarrow{\hat{m}}\|=(1,0) ; \vec{x}, \overrightarrow{x_{0}} \in \mathbb{R}_{1}^{3}, \vec{x} \text { spacelike }\right\},
$$

it must be that

$$
\hat{h}(s)=\frac{d \hat{h}}{d \hat{s}}=\frac{d^{2} \hat{h}}{d \hat{s}^{2}}=0 .
$$

This equations give us $<\overrightarrow{\hat{x}}-\overrightarrow{\hat{m}}, \overrightarrow{\hat{t}}>=0$ and $<\overrightarrow{\hat{x}}-\overrightarrow{\hat{m}}, \overrightarrow{\hat{n}}>=-\varepsilon_{\overrightarrow{\hat{t}}} \hat{\rho}$, (where $\hat{\rho}=\frac{1}{\hat{\varkappa}}$ is radius of curvature of $\left.\hat{x}(s)\right)$. On the other hand, it can be written that

$$
\overrightarrow{\hat{x}}-\overrightarrow{\hat{m}}=\hat{m}_{1} \overrightarrow{\hat{t}}+\hat{m}_{2} \overrightarrow{\hat{n}}+\hat{m}_{3} \overrightarrow{\hat{b}}
$$

for the base $\{\overrightarrow{\hat{t}}, \overrightarrow{\hat{n}}, \overrightarrow{\hat{b}}\}$, where $\hat{m}_{i} \in \mathbb{D}, 1 \leqslant i \leqslant 3$. Thus,

$$
\hat{m}_{1}=0, \hat{m}_{2}=-\varepsilon_{\overrightarrow{\hat{t}}} \varepsilon_{\overrightarrow{\hat{n}}} \hat{\rho}, \hat{m}_{3}=\mp \sqrt{\left|\varepsilon_{\overrightarrow{\hat{b}}} \hat{r}^{2}-\varepsilon_{\overrightarrow{\hat{n}}} \varepsilon_{\overrightarrow{\hat{b}}} \frac{1}{\hat{\varkappa}^{2}}\right|}
$$


are obtained. If so,

$$
\overrightarrow{\hat{m}}=\overrightarrow{\hat{x}}-\hat{m}_{2} \overrightarrow{\hat{n}}-\hat{m}_{3} \overrightarrow{\hat{b}}
$$

is found, where $\vec{m}$ and $\hat{r}$ change as the dual Lorentzian spheres change.

Corollary 4.1. Let the curve $\hat{x}(s) \subset \mathbb{D}_{1}^{3}$ be given with the coordinate neighbourhood $(I, \hat{x})$. The centers of dual Lorentzian spheres with 3-contact points with $\hat{x}(s)$ in the point $\hat{x}(s)$ lie on straight line.

Proof. From Theorem 4.1, it is written

$$
\overrightarrow{\hat{m}}=\overrightarrow{\hat{x}}+\varepsilon_{\overrightarrow{\hat{t}}} \varepsilon_{\overrightarrow{\hat{n}}} \hat{\rho} \overrightarrow{\hat{n}}+\hat{\lambda} \overrightarrow{\hat{b}}, \hat{\lambda}=\mp \sqrt{\left|\varepsilon_{\vec{b}} \hat{r}^{2}-\varepsilon_{\overrightarrow{\hat{n}}} \varepsilon_{\vec{b}} \frac{1}{\hat{\varkappa}^{2}}\right|} .
$$

Hence, this equation with the parameter $\hat{\lambda} \in \mathbb{D}$ illustrates a straight line parallel to $\overrightarrow{\hat{b}}$ and passing through the point $\overrightarrow{\hat{c}}=\overrightarrow{\hat{x}}+\underset{\hat{t}}{\varepsilon \rightarrow \hat{n}} \hat{\rho} \hat{\hat{n}}$.

Definition 4.1. The line $\overrightarrow{\hat{m}}=\overrightarrow{\hat{x}}+\varepsilon_{\overrightarrow{\hat{t}}} \varepsilon_{\overrightarrow{\hat{n}}} \hat{\rho} \overrightarrow{\hat{n}}+\hat{\lambda} \overrightarrow{\hat{b}}$, geometric place of centers of the dual Lorentzian spheres with 3 -contact points with $\hat{x}(s)$ in the point $\hat{x}(s)$ of the curve $\hat{x}(s) \in \mathbb{D}_{1}^{3}$, is called curvature axis in the point $\hat{x}(s)$ of the curve $\hat{x}(s)$.

Definition 4.2. The point $\overrightarrow{\hat{c}}=\overrightarrow{\hat{x}}+\underset{\hat{t}}{\varepsilon \rightarrow \hat{n}} \hat{\hat{\rho}} \overrightarrow{\hat{n}}$ is called the center of curvature in the point $\hat{x}(s)$ of the curve $\hat{x}(s)$.

Definition 4.3. The sphere having 4-contact points with the curve $\hat{x}(s)$ in the point $\hat{x}(s)$ of the curve $\hat{x}(s)$ is called dual Lorentzian osculating sphere or dual Lorentzian curvature sphere.

Theorem 4.2. Let the curve $\hat{x}(s) \subset \mathbb{D}_{1}^{3}$ be given with coordinate neighbourhood $(I, \hat{x})$. If $\overrightarrow{\hat{m}}$ is center of the dual Lorentzian osculating sphere in the point $\hat{x}(s)$, it is

$$
\overrightarrow{\hat{m}}=\overrightarrow{\hat{x}}+\underset{\hat{t}}{\varepsilon_{\overrightarrow{\hat{n}}}} \hat{\rho} \overrightarrow{\hat{n}}+\varepsilon_{\overrightarrow{\hat{t}}} \varepsilon_{\overrightarrow{\hat{b}}} \frac{1}{\hat{\tau}} \frac{d \hat{\rho}}{d \hat{s}} \overrightarrow{\hat{b}}
$$

Proof. Let $\overrightarrow{\hat{m}}$ be the center of the dual Lorentzian osculating sphere with 4 -contact points with the curve $\hat{x}(s)$ and $\hat{r}$ be the radius. Let us take the function 


$$
\begin{aligned}
\hat{h}: I & \rightarrow \mathbb{D} \\
s & \rightarrow \hat{h}(s)=|<\overrightarrow{\hat{x}}-\overrightarrow{\hat{m}}, \overrightarrow{\hat{x}}-\overrightarrow{\hat{m}}>|-\hat{r}^{2}
\end{aligned}
$$

into consideration. For the curve $\hat{x} \overrightarrow{(s)}$ to have 4-contact points with the dual Lorentzian sphere of

$$
\hat{S}_{1}^{2}=\left\{\overrightarrow{\hat{x}}=\vec{x}+\xi \overrightarrow{x_{0}} \mid\|\overrightarrow{\hat{x}}-\overrightarrow{\hat{m}}\|=(r, 0) ; \vec{x}, \overrightarrow{x_{0}} \in \mathbb{R}_{1}^{3}, \vec{x} \text { spacelike }\right\},
$$

it must be that

$$
\hat{h}(s)=\frac{d \hat{h}}{d \hat{s}}=\frac{d^{2} \hat{h}}{d \hat{s}^{2}}=\frac{d^{3} \hat{h}}{d \hat{s}^{3}}=0 .
$$

Thus, from this equations,

$$
\begin{gathered}
<\overrightarrow{\hat{x}}-\overrightarrow{\hat{m}}, \overrightarrow{\hat{t}}>=0, \\
<\overrightarrow{\hat{x}}-\overrightarrow{\hat{m}}, \overrightarrow{\hat{n}}>=-\varepsilon_{\overrightarrow{\hat{t}}} \hat{\rho},
\end{gathered}
$$

and

$$
\hat{\tau}<\overrightarrow{\hat{x}}-\overrightarrow{\hat{m}}, \overrightarrow{\hat{b}}>=-\varepsilon \overrightarrow{\hat{t}} \frac{d \hat{\rho}}{d \hat{s}}
$$

are obtained. Then, in the form of the base $\{\overrightarrow{\hat{t}}, \overrightarrow{\hat{n}}, \overrightarrow{\hat{b}}\}$, the center of dual Lorentzian osculating sphere can be written as

$$
\overrightarrow{\hat{x}}-\overrightarrow{\hat{m}}=\hat{\alpha} \overrightarrow{\hat{t}}+\hat{\beta} \overrightarrow{\hat{n}}+\hat{\partial} \overrightarrow{\hat{b}},
$$

where $\hat{\alpha}, \hat{\beta}$ and $\hat{\partial}$ are dual numbers. By the aid of the equations (4.2), (4.3) and (4.4), the dual numbers $\hat{\alpha}, \hat{\beta}$ and $\hat{\partial}$ are found as

$$
\hat{\alpha}=0, \hat{\beta}=-\varepsilon_{\vec{t}} \varepsilon_{\hat{n}} \hat{\rho}, \text { and } \hat{\partial}=-\varepsilon_{\vec{t}} \varepsilon \rightarrow \frac{1}{\hat{b}} \frac{d \hat{\tau}}{\frac{\hat{\rho}}{d \hat{s}}} .
$$

Thus, we see that the center of the dual Lorentzian osculating sphere is uniquely determined by

$$
\overrightarrow{\hat{m}}=\overrightarrow{\hat{x}}+\varepsilon_{\overrightarrow{\hat{t}}} \varepsilon_{\overrightarrow{\hat{n}}} \hat{\rho} \overrightarrow{\hat{n}}+\varepsilon_{\overrightarrow{\hat{t}}} \varepsilon_{\vec{b}} \frac{1}{\hat{\hat{\tau}}} \frac{d \hat{\rho}}{d \hat{s}} \overrightarrow{\hat{b}}
$$

Corollary 4.2. Let the curve $\hat{x}(s) \subset \mathbb{D}_{1}^{3}$ be given with coordinate neighbourhood $(I, \hat{x})$. If $\hat{r}$ is the radius of dual Lorentzian osculating sphere in the point $\hat{x}(s)$, it is 


$$
\hat{r}=\sqrt{\left|\varepsilon_{\vec{n}} \hat{\rho}^{2}+\varepsilon_{\overrightarrow{\hat{b}}}\left[\frac{1}{\hat{\tau}} \frac{d \hat{\rho}}{d \hat{s}}\right]^{2}\right|} .
$$

Proof. According to Theorem 4.2, the center of the dual Lorentzian osculating sphere in the point $\hat{x}(s)$ is

$$
\overrightarrow{\hat{m}}=\overrightarrow{\hat{x}}+\varepsilon_{\overrightarrow{\hat{t}}} \varepsilon_{\overrightarrow{\hat{n}}} \hat{\rho} \overrightarrow{\hat{n}}+\varepsilon_{\overrightarrow{\hat{t}}} \varepsilon \rightarrow \frac{1}{\hat{b}} \frac{d \hat{\tau}}{\hat{\tau}} \frac{\vec{\rho}}{d \hat{b}} .
$$

Therefore, from $|<\overrightarrow{\hat{x}}-\overrightarrow{\hat{m}}, \overrightarrow{\hat{x}}-\overrightarrow{\hat{m}}>|=\hat{r}^{2}$, we get

$$
\hat{r}=\sqrt{\left|\varepsilon_{\vec{n}} \hat{\rho}^{2}+\varepsilon_{\overrightarrow{\hat{b}}}\left[\frac{1}{\hat{\tau}} \frac{d \hat{\rho}}{d \hat{s}}\right]^{2}\right|} .
$$

Theorem 4.3. Let $\hat{S}_{1}^{2} \subset \mathbb{D}_{1}^{3}$ be a dual Lorentzian sphere with center $O$. If $\hat{x}(s) \subset \hat{S}_{1}^{2}$, dual Lorentzian osculating sphere in all of the points of the curve $\hat{x} \overrightarrow{(s)}$ is $\hat{S}_{1}^{2}$.

Proof. We suppose that the curve $\hat{x}(s)$ is given with coordinate neighbourhood $(I, \hat{x})$. According to Theorem 4.2 , the center of dual Lorentzian osculating sphere in the point $\hat{x}(s)$ is

$$
\overrightarrow{\hat{m}}=\overrightarrow{\hat{x}}+\varepsilon_{\overrightarrow{\hat{t}}} \varepsilon_{\overrightarrow{\hat{n}}} \hat{\rho} \overrightarrow{\hat{n}}+\varepsilon_{\overrightarrow{\hat{t}}} \varepsilon_{\overrightarrow{\hat{b}}} \frac{1}{\hat{\tau}} \frac{d \hat{\rho}}{d \hat{s}} \overrightarrow{\hat{b}}
$$

On the other hand, by the aid of the base $\{\overrightarrow{\hat{t}}, \overrightarrow{\hat{n}}, \overrightarrow{\hat{b}}\}, \hat{x}(s) \in \mathbb{D}_{1}^{3}$ can be written as

$$
\overrightarrow{\hat{x}}=-\varepsilon_{\hat{t}} \varepsilon_{\vec{n}} \hat{\rho} \overrightarrow{\hat{n}}-\varepsilon_{\hat{t}} \varepsilon \rightarrow \frac{1}{\hat{b}} \frac{d \hat{\tau}}{d \hat{\rho}} \overrightarrow{\hat{b}}
$$

Thus, from equation (4.5) we get $\overrightarrow{\hat{m}}=0$. So, it is $d(\overrightarrow{\hat{x}}, 0)=(r, 0)$. Therefore, dual Lorentzian osculating sphere in all of the points of the curve $\hat{x}(s)$ is $\hat{S}_{1}^{2}$.

Dual Lorentzian spherical curves are those curves satisfying the differential equation in natural coordinates

$$
\left|\varepsilon_{\vec{n}} \hat{\rho}^{2}+\varepsilon_{\vec{b}}\left[\frac{1}{\hat{\tau}} \frac{d \hat{\rho}}{d \hat{s}}\right]^{2}\right|=\hat{c}^{2}, \hat{c}=\text { any dual const. }
$$

Indeed, when a dual curve is spherical, its dual Lorentzian osculating spheres all coincide with the dual Lorentzian sphere on which the curve lies. Therefore, equation 
(4.5) holds, where according to equation (4.6) $\hat{c}$ is the radius of the dual Lorentzian osculating sphere.

The theorem below characterizes the relation between the centers and the radii of dual Lorentzian osculating spheres.

Theorem 4.4. The radius of dual Lorentzian osculating sphere is constant if and only if the centers of dual Lorentzian osculating spheres are constant.

Proof. Let the radius of dual Lorentzian osculating sphere be constant. In this case, for $\hat{\tau}$ and $\hat{\rho}$, not pure dual, and $\frac{d \hat{\rho}}{d \hat{s}} \neq 0$, if differentation of equation (4.7) is taken, it is found

$$
\underset{\hat{t}}{\varepsilon \rightarrow \hat{n}} \hat{\rho} \hat{\rho} \hat{\tau}+\underset{\hat{t}}{\varepsilon \rightarrow} \varepsilon_{\hat{b}} \frac{d}{d \hat{s}}\left[\frac{1}{\hat{\tau}} \frac{d \hat{\rho}}{d \hat{s}}\right]=0 .
$$

On the other hand, since

$$
\frac{d \overrightarrow{\hat{m}}}{d \hat{s}}=\left[\underset{\hat{t}}{\varepsilon} \varepsilon_{\hat{n}} \hat{\rho} \hat{\tau}+\varepsilon_{\hat{t}} \varepsilon_{\overrightarrow{\hat{b}}} \frac{d}{d \hat{s}}\left[\frac{1}{\hat{\tau}} \frac{d \hat{\rho}}{d \hat{s}}\right]\right] \overrightarrow{\hat{b}}
$$

according to equation (4.8), we get $\frac{d \overrightarrow{\hat{m}}}{d \hat{s}}=0$. This means that the centers of dual Lorentzian osculating spheres remain fixed.

Conversely, let the center of dual Lorentzian osculating sphere be constant. Hence, it is written $\frac{d \overrightarrow{\hat{m}}}{d \hat{s}}=0$ or

$$
\varepsilon_{\vec{t}} \varepsilon \rightarrow \hat{\hat{n}} \hat{\rho} \hat{\tau}+\underset{\hat{t}}{\varepsilon \rightarrow \vec{b}} \frac{d}{d \hat{s}}\left[\frac{1}{\hat{\tau}} \frac{d \hat{\rho}}{d \hat{s}}\right]=0 .
$$

On the other hand, since

$$
\varepsilon_{\vec{t}} \frac{\hat{\tau} \hat{r}}{\frac{d \hat{\rho}}{d \hat{s}}} \frac{d \hat{r}}{d \hat{s}}=\varepsilon_{\overrightarrow{\hat{t}}} \varepsilon_{\overrightarrow{\hat{n}}} \hat{\rho} \hat{\tau}+\varepsilon_{\overrightarrow{\hat{t}}} \varepsilon_{\vec{b}} \frac{d}{d \hat{s}}\left[\frac{1}{\hat{\tau}} \frac{d \hat{\rho}}{d \hat{s}}\right]
$$

according to the equation above $\frac{d \hat{r}}{d \hat{s}}=0$ is obtained. Thus, the radius of dual Lorentzian osculating sphere is constant.

Theorem 4.5. Let the curve $\hat{x}(s) \subset \mathbb{D}_{1}^{3}$ be given with coordinate neighbourhood $(I, \hat{x})$. We suppose that $\hat{\tau} \neq 0, \hat{\rho} \neq 0$ and $\frac{d \hat{\rho}}{d \hat{s}} \neq 0$ for $\forall s \in I$. In this case, $\hat{x}(s)$ is dual Lorentzian spherical curve if and only if the center of dual Lorentzian osculating sphere in the point $\hat{x}(s)$ is at the same point.

Proof. Let $\hat{x}(s)$ be dual Lorentzian spherical curve. Then, according to Theorem 4.3 , the proof is abvious. 
Conversely, if the center of dual Lorentzian osculating sphere in the point $\hat{x}(s)$ for $\forall s \in I$ is constant point $\overrightarrow{\hat{u}}$, with respect to Theorem 4.4, the radius of dual Lorentzian osculating sphere is also the same constant $\hat{r}$. Therefore, $d(\hat{x} \overrightarrow{(s)}, \overrightarrow{\hat{u}})=(r, 0)$ for all $\hat{x}(s)$. Thus, it is $\hat{x}(s) \subset \hat{S}_{1}^{2}$.

Now, let us give the characterization for a curve to be spherical from type of curvatures of it with the following theorem.

Theorem 4.6. Let the curve $\hat{x}(s) \subset \mathbb{D}_{1}^{3}$ be given with coordinate neighbourhood $(I, \hat{x}) . \hat{x}(\vec{s})$ with arc length parameter $s \in I$ is dual Lorentzian spherical curve if and only if

$$
\varepsilon_{\vec{t}} \varepsilon_{\overrightarrow{\hat{n}}} \hat{\rho} \hat{\tau}+\underset{\hat{t}}{\varepsilon_{\vec{b}}} \varepsilon_{\overrightarrow{\hat{b}}} \frac{d}{d s}\left[\frac{1}{\hat{\tau}} \frac{d \hat{\rho}}{d s} \frac{d s}{d \hat{s}}\right] \frac{d s}{d \hat{s}}=0 .
$$

Proof. Let $\hat{x}(s)$ be dual Lorentzian spherical curve. In this case, the center $\overrightarrow{\hat{m}}$ of dual Lorentzian osculating sphere for all $s \in I$ is constant. Thus, from Theorem 4.4 ,

$$
\varepsilon_{\hat{t}}^{\varepsilon} \underset{\hat{n}}{\vec{\rho}} \hat{\tau}+\underset{\hat{t}}{\varepsilon} \varepsilon_{\hat{b}} \frac{d}{d s}\left[\frac{1}{\hat{\tau}} \frac{d \hat{\rho}}{d s} \frac{d s}{d \hat{s}}\right] \frac{d s}{d \hat{s}}=0
$$

is obtained.

Conversely, let

$$
\varepsilon_{\vec{t}} \varepsilon_{\overrightarrow{\hat{n}}} \hat{\rho} \hat{\tau}+\underset{\hat{t}}{\varepsilon_{\vec{b}}} \varepsilon_{\overrightarrow{\hat{b}}} \frac{d}{d s}\left[\frac{1}{\hat{\tau}} \frac{d \hat{\rho}}{d s} \frac{d s}{d \hat{s}}\right] \frac{d s}{d \hat{s}}=0 .
$$

Thus, $\frac{d \overrightarrow{\hat{m}}}{d \hat{s}}=0$ is found. This implies that $\overrightarrow{\hat{m}}=$ constant. So, according to Theorem 4.5, $\hat{x}(\vec{s})$ is dual Lorentzian spherical curve.

On the other hand, since the differential equation (4.8) can be written as

$$
\varepsilon_{\vec{t}} \varepsilon_{\vec{n}} \hat{\rho} \hat{\tau}+\underset{\hat{t}}{\varepsilon \rightarrow \vec{b}} \frac{d}{d s}\left[\frac{1}{\hat{\tau}} \frac{d \hat{\rho}}{d s} \frac{d s}{d \hat{s}}\right] \frac{d s}{d \hat{s}}=0,
$$

if we put $\hat{u}(s)=\frac{d s}{d \hat{s}}=1-\xi \Delta$, the equation above is obtained as

$$
\underset{\hat{t}}{\varepsilon \rightarrow \vec{b}} \frac{d^{2} \hat{\rho}}{d s^{2}}+\underset{\hat{t}}{\varepsilon \rightarrow \vec{b}} \frac{\hat{u}^{\prime}(s)}{\hat{u}(s)} \frac{d \hat{\rho}}{d s}+\underset{\hat{t}}{\varepsilon} \varepsilon_{\vec{n}} \frac{\hat{\tau}^{2}}{\hat{u}^{2}(s)} \hat{\rho}=0 .
$$

Here, if we make a parameter change like $t=\int_{0}^{s} \frac{\hat{\tau}}{\hat{u}(s)} d s$, we obtain

$$
\frac{d^{2} \hat{\rho}}{d t^{2}}+\underset{\hat{n}}{\varepsilon} \underset{\hat{b}}{\vec{\rho}} \hat{\rho}=0
$$

There are two solutions for this differential equation with respect to causal character of the curve: 
(i) If the dual Lorentzian spherical curve is timelike, the solution of the differential equation is

$$
\hat{\rho}=\hat{a} \cos \left(\int_{0}^{s} \hat{\tau} d \hat{s}\right)+\hat{b} \sin \left(\int_{0}^{s} \hat{\tau} d \hat{s}\right),
$$

where $\hat{a}$ and $\hat{b}$ are arbitrary dual constants.

(ii) If the dual Lorentzian spherical curve is spacelike, the solution of the differential equation is

$$
\hat{\rho}=\hat{a} e^{-\int_{0}^{s} \hat{\tau} d \hat{s}}+\hat{b} e^{\int^{s} \hat{\tau} d \hat{s}} .
$$

Hence, the equations (4.9) and (4.10) give a characterization of the dual Lorentzian spherical curves.

The necessary and sufficient condition for the dual Lorentzian curve $\hat{x}(s)$ to be a dual Lorentzian spherical one, without having to assume that its torsion is nowhere pure-dual, may be given as follows:

Theorem 4.7. $\quad \hat{x}(s) \subset \mathbb{D}_{1}^{3}$ is dual Lorentzian spherical curve if and only if there is a differentiable function $\hat{f}=f+\xi f_{0}$ ( $f$ and $f_{0}$ are real functions defined on I) such that

$$
\hat{f} \hat{\tau}=\frac{d \hat{\rho}}{d \hat{s}}, \text { and } \frac{d \hat{f}}{d \hat{s}}+\underset{\hat{n}}{\varepsilon \rightarrow \vec{b}} \hat{\tau} \hat{\rho}=0
$$

Proof. Let us first assume that the dual Lorentzian curve $\hat{x}(s) \subset D_{1}^{3}$ lies in a dual Lorentzian sphere with center $\overrightarrow{\hat{m}}$. Define the differentiable function $\hat{f}$ by

$$
\hat{f}=-\varepsilon_{\vec{t}}<\overrightarrow{\hat{x}}-\overrightarrow{\hat{m}}, \overrightarrow{\hat{b}}>
$$

Then, the equation (4.4) becomes $\hat{f} \hat{\tau}=\frac{d \hat{\rho}}{d \hat{s}}$, which is (4.11) 1 . Consequently, differentiating (4.12) and making use of (4.3), we have that $\frac{d \hat{f}}{d \hat{s}}+\underset{\hat{n}}{\varepsilon_{\hat{b}}} \varepsilon_{\vec{b}} \hat{\tau} \hat{\rho}=0$, which is $(4.11)_{2}$.

Conversely, assume that $\hat{x}(s) \subset \mathbb{D}_{1}^{3}$ is a dual Lorentzian curve and that there is a differentiable function $\hat{f}$ such that

$$
\hat{f} \hat{\tau}=\frac{d \hat{\rho}}{d \hat{s}}, \text { and } \frac{d \hat{f}}{d \hat{s}}+\underset{\hat{n}}{\varepsilon \vec{b}} \hat{\tau} \hat{\rho}=0 .
$$


Let us consider the curve defined by

$$
\overrightarrow{\hat{m}}=\overrightarrow{\hat{x}}+\underset{\hat{t}}{\varepsilon \rightarrow \hat{n}} \hat{\rho} \overrightarrow{\hat{n}}+\underset{\hat{t}}{\varepsilon \rightarrow \vec{b}} \overrightarrow{\hat{f}} \overrightarrow{\hat{b}}
$$

and the function $\hat{r}(s)$ defined by

$$
\hat{r}^{2}(s)=|<\overrightarrow{\hat{x}}-\overrightarrow{\hat{m}}, \overrightarrow{\hat{x}}-\overrightarrow{\hat{m}}\rangle|=| \varepsilon_{\overrightarrow{\hat{n}}} \hat{\rho}^{2}+\varepsilon_{\vec{b}} \hat{f}^{2} \mid .
$$

If we differentiate the equations (4.13) and (4.14) and make use of the equations (3.1) and (4.11), we have that $\frac{d \overrightarrow{\hat{m}}}{d \hat{s}}=0$ and $\frac{d \hat{r}}{d \hat{s}}=0$. These show that $\overrightarrow{\hat{m}}$ and $\hat{r}(s)$ are constants. Hence, by Theorem 4.5, it follows that $\hat{x}(s)$ is dual Lorentzian spherical curve.

Now, differentiating the equation (4.9) (in this case, the curve $\vec{x}(s)$ is timelike.) and using the equation (4.11), we find that the function $\hat{f}$ is

$$
\hat{f}=-\hat{a} \sin \left(\int_{0}^{s} \hat{\tau} d \hat{s}\right)+\hat{b} \cos \left(\int_{0}^{s} \hat{\tau} d \hat{s}\right) .
$$

Similarly, if the curve $\hat{x}(s)$ is spacelike, we obtain the function $\hat{f}$ as

$$
\hat{f}=-\hat{a} e^{-\int_{0}^{s} \hat{\tau} d \hat{s}}+\hat{b} e^{\int_{0}^{s} \hat{\tau} d \hat{s}} .
$$

\section{Dual Lorentzian Geodesic TriHedron}

Let $\overrightarrow{\hat{e}}=\vec{e}+\xi \overrightarrow{e_{0}}$ be a dual unit vector in $\mathbb{D}_{1}^{3} \cdot \vec{e}$ and $\overrightarrow{e_{0}}$ may be interprated as the Plucker-vectors of an unambiguously determined line $l$ in $\mathbb{R}_{1}^{3}$ having $\vec{e}$ as its direction vector and passing through the point $\vec{p}=\vec{e} \wedge \overrightarrow{e_{0}}$. Providing $l$ with a positive sense according with the sense indicated by $\vec{e}$, this line becomes an oriented line as a spear. The vector $\overrightarrow{e_{0}}$ is called the moment of the spear (with respect to 0 ). We denote the spear by $\left(\vec{e}, \overrightarrow{e_{0}}\right)$. There exist a one-to-one correspondence between the set of all spears in three dimensional space and the set of all dual unit vectors:

$$
\left(\vec{e}, \overrightarrow{e_{0}}\right) \leftrightarrows \overrightarrow{\hat{e}}=\vec{e}+\xi \overrightarrow{e_{0}}
$$

Actually, the set of dual unit vectors representing the oriented lines is a subset of the set of dual unit vectors

$$
\left\{\overrightarrow{\hat{x}}=\vec{x}+\xi \overrightarrow{x_{0}} \mid<\vec{x}, \vec{x}>=1,<\vec{x}, \overrightarrow{x_{0}}>=0, \vec{x}, \overrightarrow{x_{0}} \in \mathbb{R}^{3}\right\}
$$


If we consider the Lorentzian space $\mathbb{R}_{1}^{3}$ instead of $\mathbb{R}^{3}$ the correspondence of E. Study mapping can be given as follows: Oriented timelike and spacelike lines in $\mathbb{R}_{1}^{3}$ may be represented by timelike and spacelike unit vectors with three-components in the dual Lorentzian space $\mathbb{D}_{1}^{3}$, respectively. A differentiable curve on the dual hyperbolic unit sphere $\hat{H}_{0}^{2}$ corresponds to a timelike ruled surface while a differentiable curve on the dual Lorentzian unit sphere $\hat{S}_{1}^{2}$ corresponds to any ruled surface. This correspondence is one-to-one and allows the geometry of Lorentzian ruled surface to be represented by the geometry of dual hyperbolic and Lorentzian spherical curves on $\hat{H}_{0}^{2}$ and $\hat{S}_{1}^{2}$, respectively [14].

Take into consideration a dual Lorentzian curve represented by $\overrightarrow{\hat{e}}=\vec{e}+\xi \overrightarrow{e_{0}}$ on the dual Lorentzian unit sphere $\hat{S}_{1}^{2}$ with the center 0 in the dual Lorentzian space $\mathbb{D}_{1}^{3}$, where $s$ is the arc length of the indicatrix $\overrightarrow{e(s)}$ and $e_{0}(s)$ is the moment of $\overrightarrow{e(s)}$ with respect to 0 . Then, $\left\langle\vec{e}^{\prime}, \vec{e}^{\prime}>=<\vec{t}, \vec{t}>=\varepsilon_{\vec{t}}\right.$, the tangent vector $\vec{t}=\vec{e}^{\prime}$ is a unit vector parallel to the tangent of the indicatrix. The equation $\vec{z} \wedge \vec{e}=\overrightarrow{e_{0}}$ has an infinity of solutions for the function $\overrightarrow{z(s)}$. If $z_{0}(s)$ is such a solution, the set of all solutions is given by $z \overrightarrow{(s)}=z_{0}(s)+\lambda(s) \overrightarrow{e(s)}$, where $\lambda$ is a real scalar function of $s$. Therefore, $<\vec{z}, \vec{e}^{\prime}>=<{\overrightarrow{z_{0}}}^{\prime}, \vec{e}^{\prime}>+\varepsilon_{t} \lambda$ is obtained. If we take into consideration $\lambda=\lambda_{0}=-\varepsilon_{\vec{t}}<\vec{z}^{\prime}, \vec{e}^{\prime}>, z_{0}(s)+\lambda_{0}(s) e(\vec{s})=c \overrightarrow{(s)}$ is the unique solution for $\overrightarrow{z(s)}$ with $<\overrightarrow{c(s)}, \overrightarrow{e(s)}>=0$. A dual curve given can be represented by

$$
\hat{e}(s)=\overrightarrow{e(s)}+\xi \overrightarrow{c(s)} \wedge \overrightarrow{e(s)} .
$$

$\vec{c}$ is unambiguously determined by the curve $\hat{e}(s)$. According the $(2.1)$, the arc length of the curve $\hat{e}(s)$ is

$$
\hat{s}=s+\xi \int_{0}^{s}<\vec{t}, \vec{e}_{0}^{\prime}>d s=s+\xi \int_{0}^{s} \Delta d s .
$$

Thus, $\frac{d \hat{s}}{d s}=1+\xi \Delta$ or $\frac{d s}{d \hat{s}}=1-\xi \Delta$. On the other hand, since $\left.<\vec{t},{\overrightarrow{e_{0}}}^{\prime}>=<c \vec{s}^{\prime} \wedge \overrightarrow{e(s)}, \vec{t}>=\Delta, \Delta \vec{t}=\varepsilon_{\vec{t}} c \overrightarrow{c s}^{\prime}\right)^{\prime} \wedge \overrightarrow{e(s)}$ is obtained. Hence,

$$
\frac{d \overrightarrow{\hat{e}}}{d \hat{s}}=\frac{d \overrightarrow{\hat{e}}}{d s} \frac{d s}{d \hat{s}}=\overrightarrow{\hat{t}}=\vec{t}+\xi \overrightarrow{t_{0}}=\vec{t}+\xi \vec{c} \wedge \vec{t}
$$

is obtained. By introducing the dual unit vector $\overrightarrow{\hat{g}}=\varepsilon_{\vec{e}} \varepsilon_{\vec{t}} \vec{e} \wedge \vec{t}=\vec{g}+\xi \overrightarrow{g_{0}}$, 
$\vec{g}=\varepsilon_{\vec{e}} \varepsilon_{\vec{t}} \vec{e} \wedge \vec{t}$ is found. Thus, by the aid of the (5.1) and (5.3), we have that

$$
\overrightarrow{\hat{g}}=\vec{g}+\xi \vec{c} \wedge \vec{g}
$$

Here, we will call $\{\overrightarrow{\hat{t}}, \vec{g}, \vec{e}\}$ the dual Lorentzian geodesic trihedron of the curve $\hat{e}(s)$. If the derivatives of Lorentzian inner products of $\overrightarrow{\hat{t}}, \overrightarrow{\hat{g}}$ and $\overrightarrow{\hat{e}}$ are taken and if the properties of the Lorentzian inner product are used, Frenet formulae for dual Lorentzian geodesic trihedron are written in the matrix form as

$$
\frac{d}{d \hat{s}}\left[\begin{array}{c}
\overrightarrow{\hat{t}} \\
\overrightarrow{\hat{g}} \\
\overrightarrow{\hat{e}}
\end{array}\right]=\left[\begin{array}{ccc}
0 & \varepsilon_{\vec{g}} \hat{\gamma} & -\varepsilon_{\overrightarrow{\hat{e}}} \varepsilon_{\vec{t}} \\
-\varepsilon_{\overrightarrow{\hat{t}}} \hat{\gamma} & 0 & 0 \\
1 & 0 & 0
\end{array}\right]\left[\begin{array}{c}
\overrightarrow{\hat{t}} \\
\overrightarrow{\hat{g}} \\
\overrightarrow{\hat{e}}
\end{array}\right] .
$$

The dual valued function $\hat{\gamma}(s)$ in (5.5) is called the geodesic curvature of the curve $\hat{e}(s)$. From the equations (5.3), (5.4) and (5.5),

$$
\hat{\gamma}=\varepsilon_{\vec{g}}[\gamma+\xi(\delta-\gamma \Delta)]
$$

is found, where $\delta=-\varepsilon_{\vec{e}}<\vec{c}^{\prime}, \vec{e}>$. Since $\Delta \vec{t}=\varepsilon_{\vec{t}} \vec{c}^{\prime} \wedge \vec{e}$, we find that

$$
\vec{c}^{\prime}=-\delta \vec{e}-\Delta \vec{g}
$$

On the other hand, since $\frac{d^{2} \overrightarrow{\hat{e}}}{d \hat{s}^{2}}=\frac{d \overrightarrow{\hat{t}}}{d \hat{s}}=\hat{\varkappa} \overrightarrow{\hat{n}}=\varepsilon_{\overrightarrow{\hat{g}}} \hat{\gamma} \overrightarrow{\hat{g}}-\varepsilon_{\overrightarrow{\hat{e}}} \varepsilon_{\overrightarrow{\hat{t}}} \overrightarrow{\hat{e}}$

$$
\hat{\varkappa}=\sqrt{\left|\sum_{\vec{n}} \varepsilon_{\overrightarrow{\hat{g}}} \hat{\gamma}^{2}+\varepsilon_{\overrightarrow{\hat{e}}}\right|}
$$

is obtained. Thus, the dual radius of curvature is found $\hat{\rho}=\frac{1}{\hat{\varkappa}}=\frac{1}{\sqrt{\left|\sum_{\overrightarrow{\hat{n}}} \varepsilon_{\overrightarrow{\hat{g}}} \hat{\gamma}^{2}+\varepsilon_{\overrightarrow{\hat{e}}}\right|} \mid}$.

The natural trihedron $\{\overrightarrow{\hat{t}}, \overrightarrow{\hat{n}}, \overrightarrow{\hat{b}}\}$ and the dual geodesic trihedron $\{\overrightarrow{\hat{t}}, \overrightarrow{\hat{g}}, \overrightarrow{\hat{e}}\}$ have one common axis $\overrightarrow{\hat{t}}$ so that a single dual angle $\hat{\theta}=\theta+\xi \theta_{0}$ specifies completely their relative position. For timelike vector $\overrightarrow{\hat{t}}$ and the angle $\hat{\theta}=\theta+\xi \theta_{0}$ between $\overrightarrow{\hat{n}}$ and $\vec{g}$,

$$
\left[\begin{array}{c}
\overrightarrow{\hat{t}} \\
\overrightarrow{\hat{g}} \\
\overrightarrow{\hat{e}}
\end{array}\right]=\left[\begin{array}{ccc}
1 & 0 & 0 \\
0 & \cos \hat{\theta} & \sin \hat{\theta} \\
0 & -\sin \hat{\theta} & \cos \hat{\theta}
\end{array}\right]\left[\begin{array}{c}
\overrightarrow{\hat{t}} \\
\overrightarrow{\hat{n}} \\
\overrightarrow{\hat{b}}
\end{array}\right]
$$


can be written. On the other hand, if the equations (3.1) and (5.5) are used and if the derivative of the equation $\vec{g}=\cos \hat{\theta} \vec{n}+\sin \hat{\theta} \overrightarrow{\hat{b}}$ with respect to $\hat{s}$ is taken,

$$
\hat{\rho}=\sin \hat{\theta}, \quad \hat{\gamma}=\hat{\varkappa} \cos \hat{\theta}, \quad \frac{d \hat{\theta}}{d \hat{s}}=-\hat{\tau}
$$

is found. Since $\frac{d \hat{\theta}}{d \hat{s}}=-\hat{\tau}$,

$$
\hat{\theta}(s)=\hat{\theta}(0)-\int_{0}^{s} \hat{\tau} d \hat{s}
$$

From the equations (5.5), (5.8), (5.9) and (5.10), the derivatives of the dual Lorentzian geodesic trihedron with respect to the terms of Frenet trihedron are obtained in the matrix form as

$$
\frac{d}{d \hat{s}}\left[\begin{array}{c}
\overrightarrow{\hat{t}} \\
\overrightarrow{\hat{g}} \\
\overrightarrow{\hat{e}}
\end{array}\right]=\left[\begin{array}{lll}
0 & \hat{\varkappa} & 0 \\
\hat{\gamma} & 0 & 0 \\
1 & 0 & 0
\end{array}\right]\left[\begin{array}{c}
\hat{t} \\
\overrightarrow{\hat{n}} \\
\overrightarrow{\hat{b}}
\end{array}\right] .
$$

Also, from the equations (2.1), (5.9), (5.10) and (5.8), it is seen that

$$
\frac{d}{d \hat{s}}\left[\begin{array}{c}
\overrightarrow{\hat{t}} \\
\overrightarrow{\hat{n}} \\
\overrightarrow{\hat{b}}
\end{array}\right]=\left[\begin{array}{lll}
0 & \hat{\gamma} & 1 \\
\hat{\varkappa} & \hat{\tau} \hat{\rho} & \hat{\tau} \hat{\rho} \hat{\gamma} \\
0 & \hat{\tau} \hat{\rho} \hat{\gamma} & \hat{\tau} \hat{\rho}
\end{array}\right]\left[\begin{array}{c}
\overrightarrow{\hat{t}} \\
\overrightarrow{\hat{g}} \\
\overrightarrow{\hat{e}}
\end{array}\right] .
$$

From the equations $(5.10)_{1},(5.10)_{2}$ and (5.8),

$$
\sin \hat{\theta}=\frac{1}{\sqrt{\hat{\gamma}^{2}+1}}, \quad \cos \hat{\theta}=\frac{\hat{\gamma}}{\sqrt{\hat{\gamma}^{2}+1}}
$$

is found. In addition, $\tan \hat{\theta}=-\frac{1}{\hat{\gamma}}$ is obtained. If the derivative of this equation is taken,

$$
\hat{\tau}=-\frac{d}{d \hat{s}}(\arctan \hat{\gamma}(s))
$$

is obtained.

Now, to return to equation (4.9) again, the explicit characterization of a curve $\hat{x}(s)$ can be expressed as

$$
\hat{\rho}=\hat{A} \cos \hat{\theta}(s)+\hat{B} \sin \hat{\theta}(s) .
$$


Also, the function $\hat{f}$ given in (4.15) takes the form

$$
\hat{f}=\hat{A} \sin \hat{\theta}(s)-\hat{B} \cos \hat{\theta}(s),
$$

where $\hat{A}=\hat{a} \cos \hat{\theta}(0)+\hat{b} \sin \hat{\theta}(0)$ and $\hat{B}=\hat{a} \sin \hat{\theta}(0)-\hat{b} \cos \hat{\theta}(0)$. Since the dual Lorentzian sphere is unit,

$$
1=\hat{A}^{2}+\hat{B}^{2}=\hat{a}^{2}+\hat{b}^{2}
$$

is obtained from (4.14).

This time, the curve given be spacelike. Let us show the hyperbolic angle between the vectors $\overrightarrow{\hat{e}}$ and $\overrightarrow{\hat{b}}$ as $\hat{\theta}=\theta+\xi \theta_{0}$. In this situation, the formulae

$$
\left[\begin{array}{c}
\overrightarrow{\hat{t}} \\
\overrightarrow{\hat{g}} \\
\overrightarrow{\hat{e}}
\end{array}\right]=\left[\begin{array}{ccc}
1 & 0 & 0 \\
0 & \cosh \hat{\theta} & \sinh \hat{\theta} \\
0 & \sinh \hat{\theta} & \cosh \hat{\theta}
\end{array}\right]\left[\begin{array}{c}
\overrightarrow{\hat{t}} \\
\overrightarrow{\hat{n}} \\
\overrightarrow{\hat{b}}
\end{array}\right]
$$

can be written. If the equations (3.1) and (5.5) are used and if the derivative of the equation $\overrightarrow{\hat{g}}=\cosh \hat{\theta} \vec{n}+\sinh \hat{\theta} \vec{b}$ with respect to $\hat{s}$ is taken,

$$
\hat{\rho}=\sinh \hat{\theta}, \quad \hat{\gamma}=\hat{\varkappa} \cosh \hat{\theta}, \text { and } \frac{d \hat{\theta}}{d \hat{s}}=-\hat{\tau}
$$

are obtained. On the other hand, since $\frac{d \hat{\theta}}{d \hat{s}}=-\hat{\tau}$, it is

$$
\hat{\theta}(s)=\hat{\theta}(0)-\int_{0}^{s} \hat{\tau} d \hat{s} .
$$

According to the equations (5.5), (5.8), (5.18) and (5.19), the derivatives of the dual Lorentzian geodesic trihedron with respect to the terms of Frenet trihedron are obtained in the matrix form as

$$
\frac{d}{d \hat{s}}\left[\begin{array}{c}
\overrightarrow{\hat{t}} \\
\overrightarrow{\hat{g}} \\
\overrightarrow{\hat{e}}
\end{array}\right]=\left[\begin{array}{lll}
0 & \hat{\varkappa} & 0 \\
-\hat{\gamma} & 0 & 0 \\
1 & 0 & 0
\end{array}\right]\left[\begin{array}{c}
\overrightarrow{\hat{t}} \\
\overrightarrow{\hat{n}} \\
\overrightarrow{\hat{b}}
\end{array}\right] .
$$

Hence, if we take into consideration the equations (3.1), (5.18), (5.19) and (5.8), we obtain the formula

$$
\frac{d}{d \hat{s}}\left[\begin{array}{c}
\overrightarrow{\hat{t}} \\
\overrightarrow{\hat{n}} \\
\overrightarrow{\hat{b}}
\end{array}\right]=\left[\begin{array}{lll}
0 & \hat{\gamma} & -1 \\
-\hat{\varkappa} & -\hat{\tau} \hat{\rho} & \hat{\tau} \hat{\rho} \hat{\gamma} \\
0 & \hat{\tau} \hat{\rho} \hat{\gamma} & -\hat{\tau} \hat{\rho}
\end{array}\right]\left[\begin{array}{c}
\overrightarrow{\hat{t}} \\
\overrightarrow{\hat{g}} \\
\overrightarrow{\hat{e}}
\end{array}\right] .
$$


From the equations $(5.19)_{1},(5.19)_{2}$ and (5.8), it follows that

$$
\sinh \hat{\theta}=\frac{1}{\sqrt{\left|\hat{\gamma}^{2}-1\right|}}, \quad \cosh \hat{\theta}=\frac{\hat{\gamma}}{\sqrt{\left|\hat{\gamma}^{2}-1\right|}}
$$

In addition, $\tanh \hat{\theta}=\frac{1}{\hat{\gamma}}$ is obtained. If we differentiate of this equation, we find that

$$
\hat{\tau}=\frac{1}{\left|\hat{\gamma}^{2}-1\right|} \frac{d \hat{\gamma}}{d \hat{s}}
$$

Now, to return to equation (4.10) again, the explicit characterization of a dual Lorentzian curve $\hat{x}(\vec{s})$ can be expressed as below

$$
\hat{\rho}=\hat{a} e^{-(\hat{\theta}(0)-\hat{\theta}(s))}+\hat{b} e^{(\hat{\theta}(0)-\hat{\theta}(s))} .
$$

On the other hand, the function $\hat{f}$ given in (4.16) is written

$$
\hat{f}=-\hat{a} e^{-(\hat{\theta}(0)-\hat{\theta}(s))}+\hat{b} e^{(\hat{\theta}(0)-\hat{\theta}(s))} .
$$

Since the dual Lorentzian sphere is unit, $1=|4 \hat{a} \hat{b}|$ is found from equation (4.14).

Remark 5.1. The properties given in this study are valid for dual hyperbolic space in the dual Lorentzian space, too.

\section{REFERENCES}

1. K. Akutagawa and S. Nishikawa, The Gauss Map and Spacelike Surfaces with Prescribed Mean Curvature in Minkowski 3-Space, Tohoku Math. J., 42 (1990), 67-82.

2. S. Birman and K. Nomizu, Trigonometry in Lorentzian Geometry, Am. Math. Mon., 91 (1984), 543-549.

3. W. Blaschke, Vorlesungen Über Differential Geometry I., Verlag von Julieus Springer in Berlin, 1930, pp. 89.

4. S. Breuer and D. Gottlieb, Explicit Characterization of Spherical Curves, Proc. Am. Math. Soc., 27 (1971), 126-127.

5. W. K. Clifford, Preliminary Sketch of Biquaternions, Proceedings of London Math. Soc., 4 (1873), 361-395.

6. A. C. Çöken, Joachimsthal Theorem for Hypersurfaces in the Semi-Euclidean Spaces, Doctoral Dissertation, Osmangazi University Graduate School of Natural and Applied Science Department of Mathematics, Eskişehir, 1995.

7. H. H. Hacısalihog̃lu, Acceleration Axes in Spatial Kinamatics I., Communications, Série A: Mathématiques, Physique et Astronomie, Tome 20A, (1971), 1-15. 
8. W. Guggenheimer, Differential Geometry, McGraw-Hill, New York, 1963.

9. Ö. Köse, Ş. Nizamoglu and M. Sezer, An Expilicit Characterization of Dual Spherical Curves, Doga Mat., 12(3) (1998), 105-113.

10. B. O"Neill, Semi-Riemannian Geometry with Applications to relativity, Academic press Inc, London, 1983.

11. E. Study, Geometrie der Dynamen, Leipzig, 1903.

12. A. C. Thompson, Minkowski Geometry, Cambridge University Press, 1996.

13. M. P. Torgasev and E. Sucurovic, Some Characterizations of the Lorentzian Spherical Timelike and Null Curves, Matematicki Vesnik, 53 (2001), 21-27.

14. H. H. Uğurlu and A. Çalışkan, The Study Mapping for Directed Spacelike and Timelike Lines in Minkowski 3-space $\mathbb{R}_{1}^{3}$, Mathematical \& Computational Applications, 1(2) (1996), 142-148.

15. Y. C. Wong, On an Explicit Characterization of Spherical Curves, Proc. Am. Math. Soc., 34 (1972), 239-242.

16. Y. Wong, A Global Formulation of the Condition for a Curve to Lie in a Sphere, Monatsh. Math., 67 (1963), 363-365.

Nihat Ayylldı, A. Ceylan Çơken and Ahmet Yucesan

Department of Mathematics,

Süleyman Demirel University, 32260, Isparta, Turkey

E-mail: ayyildiz@fef.sdu.edu.tr

E-mail: ceylan@fef.sdu.edu.tr

E-mail: yucesan@fef.sdu.edu.tr 